\title{
A Modeling and Assessing Method Based on Bayesian Networks
}

\author{
Zhi Liu and Jie Jiang \\ Software College, Zhejiang University of Technology \\ Hangzhou, China, 310032 \\ lzhi@zjut.edu.cn
}

\begin{abstract}
In order to give adaptive instruction to the learner, it needs to know what knowledge the learner has and what goals the learner is trying to archive. This paper proposes a method to build a model based on Bayesian networks in order to assess the learner's knowledge level. An overlay student model based on Bayesian networks is presented. This student model built on knowledge relationships with prediction ability is discussed in details. In this model, an assessing method based on Logistic model in IRT with three parameters is provided to evaluate student's performance. A case study in the course of Data Structure is illustrated in this paper.
\end{abstract}

Keywords: Modeling, Assessing, Bayesian networks.

\section{Introduction}

In the e-learning field, many researchers have focused on how to instruct the learners in order to support them adaptive learning. Because the learners' backgrounds are different and what goals they are trying to archive are different, they need to be instructed individually in the e-learning system. In order to assess the learner, we propose an effective student modeling method using overlay model to represent the beliefs for the student's knowledge. In this model, the student's knowledge is regarded as the subset of expert's knowledge. The expert's knowledge can be divided into various knowledge items in different levels. The student model is used to represent the understanding degrees on those knowledge items. The teaching goal is to reduce the difference between the expert's knowledge and student's knowledge.

\section{Student Modeling Method}

In this paper, we analyze and build the student model in the course of Data Structure. Each type of data structures has its various applications based on its characteristics. Here, we describe the various knowledge items in the unit of Binary Tree in table 1.

There are two kinds of relationships between knowledge items: organization relationships and dependency relationships. Combining these two structures, we can build the student model based on knowledge relationships in figure 1. 
Table 1. The knowledge items in the unit of Binary Tree

\begin{tabular}{|c|c|c|c|}
\hline Node & Knowledge item & Node & Knowledge item \\
\hline BTKU & Binary Tree Knowledge Unit & BTT & Binary Tree Traversal \\
\hline BTC & Binary Tree Concept & LT & Binary Tree Level Traversal \\
\hline BTE & Binary Tree Establish & LA & Level Traversal Application \\
\hline CBT & Complete Binary Tree & PIPT & Pre/In/Post order Traversal \\
\hline BTCH & Binary Tree Character & PIPA & Pre/In/Post order Traversal App \\
\hline BTD & Binary Tree Definition & QUU & Queue Knowledge Unit \\
\hline BTSS & Binary Tree Sequential Storage & STU & Stack Knowledge Unit \\
\hline BTCS & Binary Tree Chained Storage & CHU & Chain Knowledge Unit \\
\hline
\end{tabular}

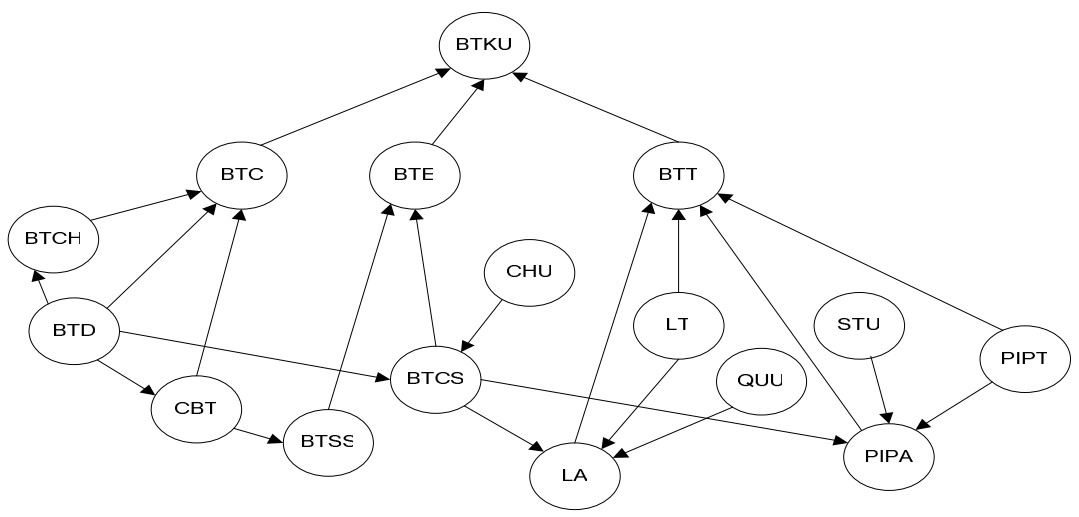

Fig. 1. The student model based on knowledge relationships

In the organization relationships, the item at the beginning of an arrow is the parent item to the item at the end point. The items in the topological structure have different importance levels and difficulty levels. They also have different influences to their parent items. We define the different weights of the knowledge item $\mathrm{I}_{\mathrm{i}}$ to influence its parent in different status: High, Medium, Low, and UMaster. For each item $\mathrm{I}_{\mathrm{i}}$, its weight to its parent is defined as $\mathrm{w}_{\mathrm{i}}$. The $\theta$ is defined as $\theta_{I_{\mathrm{i}}} \in\{$ High,Medium,Low,UMaster $\}$. Its updated weigh will be given in formula 1 :

$$
w_{i}^{\prime}=\left\{\begin{array}{lll}
w_{i} & \text { if } & \theta_{I_{i}}=\text { High } \\
\frac{2}{3} w_{i} & \text { if } & \theta_{I_{i}}=\text { Medium } \\
\frac{1}{3} w_{i} & \text { if } & \theta_{I_{i}}=\text { Low } \\
0 & \text { if } & \theta_{I_{i}}=\text { UMaster }
\end{array}\right.
$$

Under the expert's help, we could decide the weight distributions. So we could calculate the conditional probability among the items in the organization relationships. The dependency relationships in the student model could be used to predict the related mastered status based on status of other item. Here, we use the 
parameter learning algorithm, which was proposed by Heckerman[2], to identify these conditional probabilities. The hypothesis is described in the formula 2 :

$$
p\left(K_{N+1} \mid D_{s}, S^{s}\right)=\prod_{i=1}^{n} \frac{\alpha_{i j k}+N_{i j k}}{\alpha_{i j}+N_{i j}}
$$

In which $\alpha_{i j}=\sum_{k=1}^{r_{i}} \alpha_{i j k}, \quad N_{i j}=\sum_{k=1}^{r_{i}} N_{i j k}$. Where $\mathrm{N}_{\mathrm{i}, \mathrm{k} k}$ is the number of cases in domain D, in which $X_{i}={ }_{x_{i}}{ }^{k}$ and $\mathrm{Pa}_{i}=\mathrm{pa}_{i}{ }^{j}$. Here, we use $\mathrm{D}_{\mathrm{s}}$ as the student assessing sample. $S^{S}$ is the topological structure in the student model. $K_{\mathrm{N}+1}$ is some knowledge item.

\section{Assessing Method}

Here we add the testing nodes in the student model in order to get the student's responses as evidences[3]. The assessing parameter is the prior probability between the knowledge item and testing node and can be described by the item characteristic curve(ICC) with three parameters[1]. The model can be written as formula 3:

$$
P(T=1 \mid I=\theta)=P(\theta)=c_{i}+\left(1-c_{i}\right) \frac{1}{1+e^{-1.7 a_{i}\left(\theta-b_{i}\right)}}
$$

In which, the parameter $\mathrm{a}, \mathrm{b}$ and $\mathrm{c}$ could be decided by the experts. We use four statuses, such as $\mathrm{H}, \mathrm{M}, \mathrm{L}$, and UMaster. They are defined as $\mathrm{P}\left(\theta_{\mathrm{H}}\right)=1-\mathrm{s}, \mathrm{P}\left(\theta_{\mathrm{M}}\right)=2 *\left(\theta_{\mathrm{H}}\right.$ - $\left.\theta_{\mathrm{U}}\right) / 3, \mathrm{P}\left(\theta_{\mathrm{L}}\right)=\left(\theta_{\mathrm{H}}-\theta_{\mathrm{U}}\right) / 3$, and $\mathrm{P}\left(\theta_{\mathrm{U}}\right)=\mathrm{c}$. Here $\mathrm{s}$ is the probability of misplay. Then we could predigest the formula as formula 4 :

$$
\left\{\begin{array}{l}
\theta_{H}=\frac{\log \frac{s}{1-s-c_{i}}}{-1.7 a_{i}}+b_{i} \\
\theta_{M}=\frac{2}{3} \theta_{H} \\
\theta_{L}=\frac{1}{3} \theta_{H}
\end{array}\right.
$$

So we could assess the student's ability according to his answer with this assessing method. The student model can be described as the set of KnowledgeItem-Value, in which the KnowledgeItem is the knowledge unit, and Value is the examinee's mastery level $\theta$. The value of $\theta$ belongs to the range of (High, Medium, Low, UMaster). So we can calculate the value of meta-knowledge item $I_{a}$ in the formula 5:

$$
V_{I \mathrm{a}}=\theta \quad \text { if } \quad \exists P\left(I_{a}=\theta^{\prime}\right)<P\left(I_{a}=\theta\right)
$$

In which $\theta^{\prime} \in\{\mathrm{S}-\theta\}, \mathrm{S}=\{$ High, Medium, Low, UMaster $\}$. Here, we define the maximal marginal probability (MP) of the student's knowledge level as the student's mastery ability. $\left\{I_{a}\right\}$ represents the whole knowledge items included in the composed knowledge item $\mathrm{I}_{\mathrm{o}}$ and minMP is the minimum marginal probability. We can calculate the value of the composed knowledge item in formula 6 : 


$$
\theta_{I_{\mathrm{o}}}= \begin{cases}\text { High } & \text { minMP }+2 / 3(1-\min M P)<P\left(I_{\mathrm{o}}=\text { Master }\right)<1 \\ \text { Medium } & 2 / 3 \min M P+1 / 3(1-\min M P)<P\left(I_{\mathrm{o}}=\text { Master }\right)<\min M P+2 / 3(1-\min M P) \\ \text { Low } & 1 / 3 \min M P<P\left(I_{\mathrm{o}}=\text { Master }\right)<2 / 3 \min M P+1 / 3(1-\min M P) \\ \text { UMaster } & 0<P\left(I_{\mathrm{o}}=\text { Master }\right)<1 / 3 \min M P\end{cases}
$$

So we can get the final student model based on the marginal probability (MP) coming from the updated Bayesian networks.

\section{Conclusions}

In this paper, we propose an effective student modeling method built on organization relationships and dependency relationships among the knowledge items based on Bayesian networks. The dependency relationships among the knowledge items are reflected in this overlay model. In order to assess student's performance, we adopt Logistic model with three parameters in IRT model to calculate the conditional probability distribution among the testing items. In the further work, we will improve and apply this method in the whole process of the course of Data Structure.

\section{References}

1. Collins, J.A.,Greer, J.E., Huang, S.X.: Adaptive Assessment Using Granularity Hierarchies and Bayesian Nets. In: Frasson, C., Gauthier, G., Lesgold, A. (eds.): Intelligent Tutoring Systems. Third International Conference, ITS '96, Canada, Proceedings. Lecture Notes in Computer Science, Vol. 1086. Springer(1996) 569-577

2. Heckerman, D.: A Tutorial on Learning with Bayesian Networks. In: Jordan, M. (ed.): Learning in Graphical Models. MIT Press, Cambridge, MA (1999)

3. Hambleton, R.K.: Principles and Selected Applications of Item Response Theory. In: Linn, R. L. (ed.): Educational measurement (3rd edn.). New York: American Council on Education and Macmillan (1989) 147-200 